\title{
Cyndi: a multi-objective evolution algorithm based method for bioactive molecular conformational generation Xiaofeng Liu'1, Fang Bai ${ }^{2,4}$, Sisheng Ouyang ${ }^{1}$, Xicheng Wang ${ }^{2}$, Honglin $\mathrm{Li}^{* 1,2,3}$ and Hualiang Jiang*1,3
}

Address: ${ }^{1}$ Drug Discovery and Design Center, State Key Laboratory of Drug Research, Shanghai Institute of Materia Medica, Chinese Academy of Sciences, Shanghai 201203, PR China, ${ }^{2}$ Department of Engineering Mechanics, State Key Laboratory of Structural Analysis for Industrial Equipment, Dalian University of Technology, Dalian 116023, PR China, ${ }^{3}$ School of Pharmacy, East China University of Science and Technology, Shanghai 200237, PR China and ${ }^{4}$ Department of Chemical Engineering, Dalian University of Technology, Dalian 116012, PR China

Email: Xiaofeng Liu -xfliu@mail.shcnc.ac.cn; Fang Bai - fang_bai@live.cn; Sisheng Ouyang - youngseason@yahoo.cn; Xicheng Wang - guixum@dlut.edu.cn; Honglin Li* - hlli@mail.shcnc.ac.cn; Hualiang Jiang* - hljiang@mail.shcnc.ac.cn

* Corresponding authors

Published: 3I March 2009

BMC Bioinformatics 2009, 10:101 doi:10.1186/1471-2105-10-101
Received: 27 August 2008

Accepted: 31 March 2009

This article is available from: http://www.biomedcentral.com/I47/-2/05/I0/I0I

(c) 2009 Liu et al; licensee BioMed Central Ltd.

This is an Open Access article distributed under the terms of the Creative Commons Attribution License (http://creativecommons.org/licenses/by/2.0), which permits unrestricted use, distribution, and reproduction in any medium, provided the original work is properly cited.

\begin{abstract}
Background: Conformation generation is a ubiquitous problem in molecule modelling. Many applications require sampling the broad molecular conformational space or perceiving the bioactive conformers to ensure success. Numerous in silico methods have been proposed in an attempt to resolve the problem, ranging from deterministic to non-deterministic and systemic to stochastic ones. In this work, we described an efficient conformation sampling method named Cyndi, which is based on multi-objective evolution algorithm.

Results: The conformational perturbation is subjected to evolutionary operation on the genome encoded with dihedral torsions. Various objectives are designated to render the generated Pareto optimal conformers to be energy-favoured as well as evenly scattered across the conformational space. An optional objective concerning the degree of molecular extension is added to achieve geometrically extended or compact conformations which have been observed to impact the molecular bioactivity $U$ Comput -Aided Mol Des 2002, I6: 105-1 I2). Testing the performance of Cyndi against a test set consisting of 329 small molecules reveals an average minimum RMSD of $0.864 \AA$ to corresponding bioactive conformations, indicating Cyndi is highly competitive against other conformation generation methods. Meanwhile, the high-speed performance $(0.49 \pm 0.18$ seconds per molecule) renders Cyndi to be a practical toolkit for conformational database preparation and facilitates subsequent pharmacophore mapping or rigid docking. The copy of precompiled executable of Cyndi and the test set molecules in mol2 format are accessible in Additional file I.

Conclusion: On the basis of MOEA algorithm, we present a new, highly efficient conformation generation method, Cyndi, and report the results of validation and performance studies comparing with other four methods. The results reveal that Cyndi is capable of generating geometrically diverse conformers and outperforms other four multiple conformer generators in the case of reproducing the bioactive conformations against 329 structures. The speed advantage indicates Cyndi is a powerful alternative method for extensive conformational sampling and large-scale conformer database preparation.
\end{abstract}




\section{Background}

One of the imperative aspects in drug design and development is to perceive corresponding bioactive conformations which determine the physical and biological properties of drugs [1]. Conformation generation is the kernel in computer-aided drug design (CADD) methods such as molecular docking [2-4], pharmacophore construction and matching $[5,6], 3 \mathrm{D}$ database searching [79], 3D-QSAR [10-12], and molecular similarity/dissimilarity analysis [13], to name a few. The ability to account for conformational flexibility is highly valued by these methods as it presumes that small molecules have to adopt energy-reasonable conformations in respect of different environments. However, according to Boltzmann Law, the properties observed for "one molecule" are actually the conformer-ensemble averages [14]. The conformers with high energies contribute little to the ensembleaverage properties quantitatively and consequently have to be discarded during the conformation generation process. To select those low-energy conformers, a brute-force method can be applied to enumerate a set of conformations to describe the real-life distribution of the molecular conformational ensemble across the energy surface. Unfortunately, thorough conformational sampling may lead to combinatorial explosion problem even if the molecules are decomposed into fragments first and recombined into new conformers using predefined torsion library [15]. Therefore, a practical conformational ensemble should guarantee the conformers are energy reasonable and can span available conformational space evenly.

Recent studies on crystal structures of ligand-protein complexes revealed that the bioactive molecules tend to adopt more extended conformations than compact ones [16] and may be several $\mathrm{kcal} / \mathrm{mol}$ higher in energy than their respective global energy minima [17]. Although our knowledge about the pharmacologically allowed conformational space is still limited, one of the criteria for accessing conformer generation tools remains to be to what extent the experimental determined conformations can be reproduced as quickly as possible since it's not applicable to cover the whole conformational space in short time. Researchers are referred to the works by Bostrom who evaluated the capability of reproducing the bioactive conformations of several state-of-art conformation generation programs [18-20]. When it comes to conformational analysis in which multiple low-energy conformations are required, the generated conformers need to be geometrically distinct in case that some "hot spots" of the conformational space are over sampled, which cannot reflect the molecular flexibility because duplicated conformers failed to provide new information about the system. From this point of view, conformation generation may be formulated as a multi-objective optimization process in which the optima are not dominated by sole criteria exclusively. Moreover, besides of potential energy and geometrical diversity restraints, other sophisticated or rule-of-thumb criteria such as pharmacophore and binding pocket mapping can be implemented to sample more biased conformers fulfilling these objectives.

As a non-deterministic optimization method, genetic algorithm (GA) has been broadly applied in molecular docking, pharmacophore construction, and conformation generation [21-31]. Most traditional GA implementations of conformation generation perturb the dihedral torsions of rotatable bonds (sometimes plus flipping the ring conformations by modifying the geometry of $\mathrm{sp}^{3}$ hybridized atoms at the junctions of two (or more) fused aliphatic rings) using single objective function, which can be either potential energy of single individual conformers or some sophisticated designed metric of conformational space completeness/saturation in conjunction with the population each individual belongs to. For a detailed review of application of single objective GA in computer-aided drug design see the references [32,33]. However, in practical application, an ideal conformer ensemble must meet both energy and diversity criteria simultaneously (even though they conflict to each other sometimes), and it's imperative to consider all the objectives to find a set of equally valid optima (namely Pareto-optimal solutions) to maintain the balance between these restraints. Consequently multiobjective genetic algorithm (MOGA) or multi-objective evolution algorithm (MOEA) is an alternative appropriate method to solve such trade-off problems.

MOGA or MOEA method has been successfully applied to diverse areas in CADD, e.g. molecular docking [34], pharmacophore generation [35,36], combinational library building [37], and QSAR analysis [38]. During the preparation of this manuscript, Vainio used the MOGA method to generate conformer ensembles for drug-size organic molecules [39]. In his work, two uncorrelated objective functions, van de Waals (VDW) energy and dihedral torsion energy, were calculated individually using the MMFF94 force field. A niche filter on the basis of calculating the root mean square deviation (RMSD) between conformers as well as a user-definable energy cutoff value were used to reduce the size of the generated ensemble in the post processing step. Tested against the CCDC/Astex test set containing 311 entries with known bioactive conformations, the authors declared their method successfully produced low-energy conformers that are geometrically distinct from each other, which was the result their method designed to obtain.

In this work, a MOEA-based conformation generation method, named Cyndi, is presented with four specially designed objective functions. The conformers are encoded into GA individuals with the dihedral torsions of rotatable 
bonds, VDW and torsional energy terms are selected as two distinctive objectives to separate the generated conformers in energy space using Tripos force field [40]; moreover, the geometric dissimilarity (GD) of the individuals is characterized by comparing the RMSD value between each individual and the corresponding initial conformer. As an optional objective, the molecular gyration radius is employed to sample the conformations with various geometric extension degrees. As a proof-of-concept test, a combined test set composed of 329 small molecules, whose bioactive conformers are available from Protein Data Bank (PDB) [41], is benchmarked against our method. The ability of Cyndi in reproducing bioactive conformations as well as generating geometrically diverse conformational ensembles is well assessed and the results demonstrate that Cyndi provides an effective means to sample the conformational space and find the set of conformers with expected energy stability and geometric diversity.

\section{Results and discussion Flowchart}

The design and application of the genomes and objectives are based on $o$-MOEA method developed by Deb et al. [42]. The general flowchart of Cyndi is elucidated in Figure 1 and summarized below:

a. Read the input molecules, and then initialize the population of $N$ individuals by perturbing the input 3D conformer with randomly generated dihedral angles. Afterwards, step through the population to pick up the non-dominant individuals into archives.

b. Pick up the parents using tournament selection to generate new child with crossover and mutation operations, calculate the fitness for the new child and update the archive if the new child dominates any individuals in the archive. Repeat the generation procedure until the termination condition is achieved.

c. Discard the geometrical redundant conformers with the RMSD filter.

d. Optimize the remaining conformers with energy minimization and discard the conformers outside the energy cutoff, then discard the geometrical redundant conformers again.

e. Output the final generated conformers.

\section{Profiles of conformer ensembles generated by MOEA method}

With traditional single-objective approach, the solutions identified through several independent runs may be local minimum in a single-objective space. Hence, compromise between energy and geometrical feasibility has to be explicitly considered to select the solutions from the single-objective conformational space [37]. Cyndi surmounted this problem by offering a set of pareto solutions from which the users can select a conformer bearing favoured VDW energy but low GD value or vice versa. In this way the Pareto frontier actually covers the different region of the solution space and suppresses the "randomness" inherited with the EA algorithm. Different conformations are generated and possess rationality with respect to at least one objective.

For the case with 2-objective functions, the Pareto optimal solutions in the final archive should distribute across a 2D Pareto frontier curve and non-overlap with each other. For the case with 3-objective functions, the Pareto front curve transformed into a 3D surface whose profile is hard to predict. Therefore, to visualize the distribution of the solutions on the Pareto front, the exemplary scatter points as well as surface landscape for the ligand of $1 \mathrm{mcr}$ (PDB ID) is plotted in the 3D space composed of 3 objective values of each solution in the final archive (see Figures $2 \mathrm{a}$ and $2 \mathrm{~b}$ ). To clarify the result, the 3D hypersurface are mapped onto the plane formed by the other two objectives in contour mode that using continuous deepening colors to represent increasing GD values (see Figure 2c). Then corresponding solutions are also mapped on the same plane as scattered points whose sizes varied with corresponding RMSD values. One can see the solutions in the final archive are separated quite well in the three-objective space and only few points overlapped on the solution surface (marked with red ovals as outliers). These solutions are the representatives of the feasible solution space.

\section{Computational Performance of Cyndi}

The ability for conformation generation of Cyndi was tested against a data set containing 329 drug-sized molecules whose bioactive conformations were extracted from the PDB database (see Methods Section). Figure 3 presents the distribution of the rotatable bonds of the test set, the PDB IDs and rotatable bonds numbers corresponding to each molecule are listed in Table A1 (see Additional file 2). Most of the molecules posses less than 11 rotatable bonds. To access the computational cost of Cyndi, the histogram of processing times is presented in Figure 4. For the overwhelming majority of the molecules, the conformation generation time distributes from 0.2 seconds to 0.6 seconds and the average computation time is about $0.49 \pm 0.18$ seconds per molecule. The computational cost of post minimization was not considered during the evaluation because energy minimization is the major time limit step for most stochastic conformational analysis algorithms and intensively depends on the applied local optimization algorithms, adopted force field, and specified minimization iterations. Alternatively the conformer 


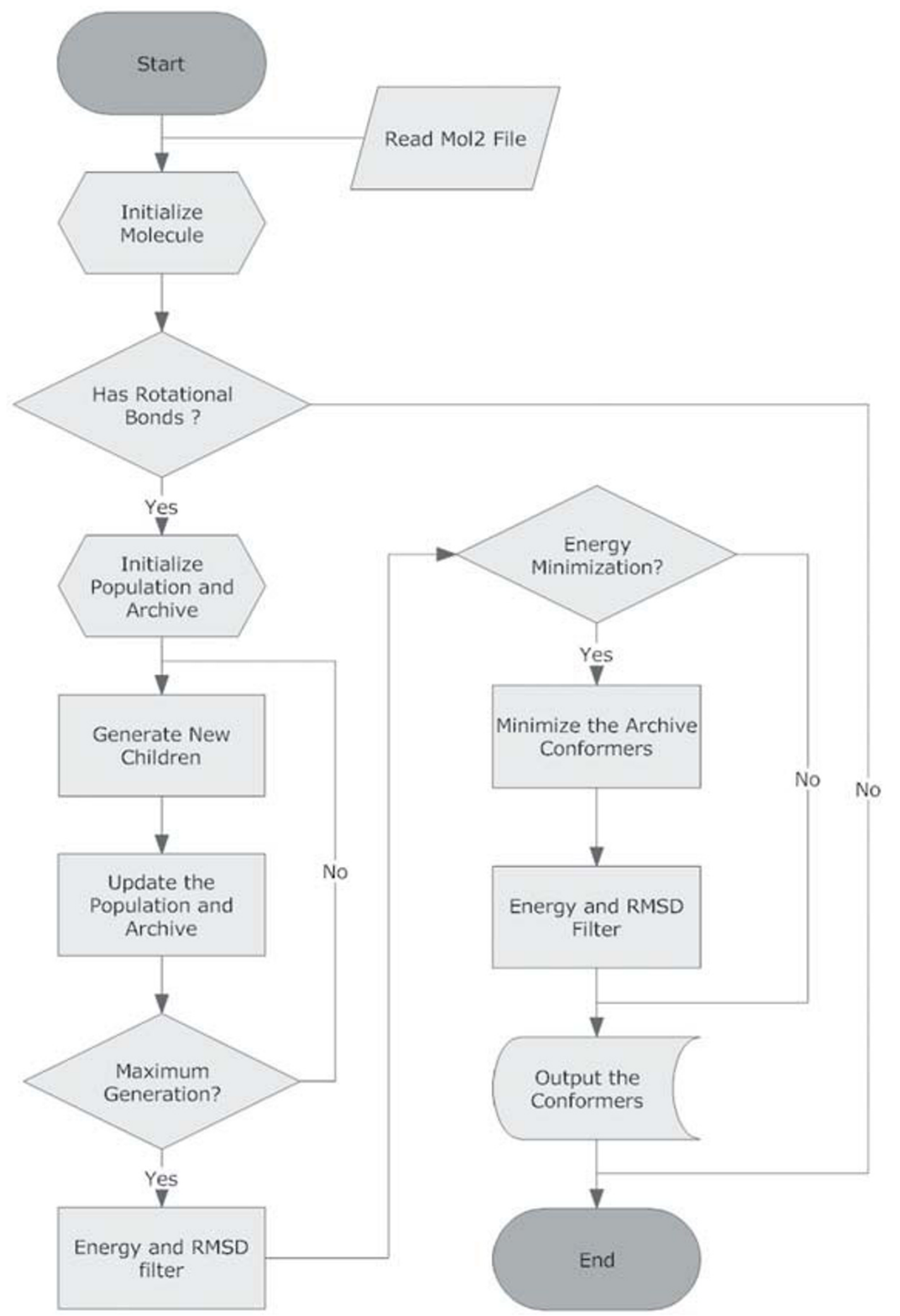

Figure I

Flowchart of Cyndi method. 


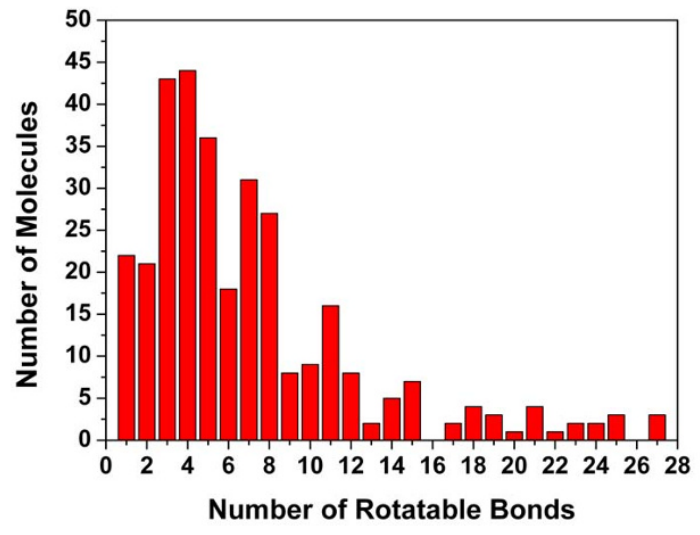

Figure 2

Profile of solution space of the final archive from the ligands of PDB entry IMCR. A total of 65 solutions are reserved in the archive. (a) 3D grid surface landscape of Pareto solutions projected on the 3-objective space. The colors are varied according to the Geometric Dissimilarity (GD, represented by RMSD to initial conformation) values as the color bar legend labelled. Three low GD valleys are labelled as the black arrows. (b) 3D scatter points representation of the Pareto solutions in 3-objective space. (c) 2D contour representation of Pareto solutions in 3-objective space. The colors are deepened as the GD values increase. The 65 conformers are projected on the space with black points, whose size varies according to their GD values respectively. The overlapped solutions are marked with red ovals as outliers.

ensembles generated with Cyndi can also be energy minimized by external softwares implemented with alternative force fields, therefore the energy optimization is discarded from computational time evaluation. As a matter of fact, the average computational time of Cyndi amounts to 23.68 seconds and never exceeds 50 seconds for each molecular conformer ensemble in an independent run, which is still acceptable and superior to that of Balloon or
Catalyst Best (at least 100 seconds per ligand conformer ensemble averagely). The result presented here proves that Cyndi is extremely fast and capable of generating conformers in nearly constant time disregarding the molecular flexibility, and therefore, can be utilized to prepare conformational database for large-scale molecular database used in virtual screening.

Cyndi is originally designed as an "upstream" conformation generator for ligand-based virtual screening in the pipeline of computer-aided drug design. The numbers of generated conformers have to be limited due to the fact that "downstream" calculations are probably highly computationally expensive. Theoretically the sizes of the conformational ensembles may increase significantly for highly flexible molecules. The number of conformers obtained after post processing are plotted against the number of rotatable bonds of each molecules (Figure 5). Interestingly, the numbers of generated conformers don't increase linearly with the numbers of rotatable bonds as expected, instead, a skewed distribution with a peak value of 7 rotatable bonds was observed (as plotted in grey line). For highly flexible molecules with more than 20 rotatable bonds, the RMSD filter scaled on number of rotatable bonds tends to drive the geometric similarity criterion more stringent by increasing RMSD tolerance. Therefore the conformational space in the archive is considered to be more "crowded" and more conformers were discarded for the highly flexible molecules. This treatment was a trade-off between elimination of conformational "combinatorial explosion" and enumeration of excessive geometrically diverse conformers. Cyndi employs fixed population size, and if one hopes to sample the conformational space for the flexible molecules more thoroughly, multiple runs or increased population size is recommended. Cyndi combines the Pareto optimal conformers from independent runs and outputs final conformation ensemble after energy as well as geometric filter. a

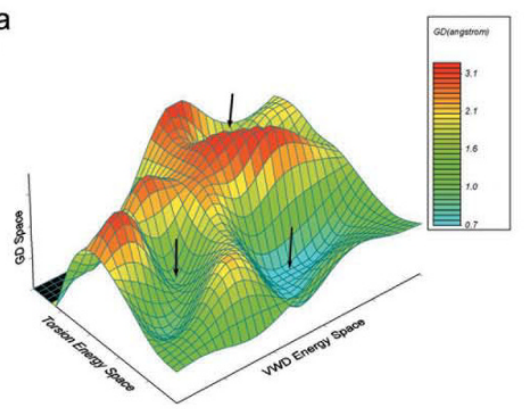

b

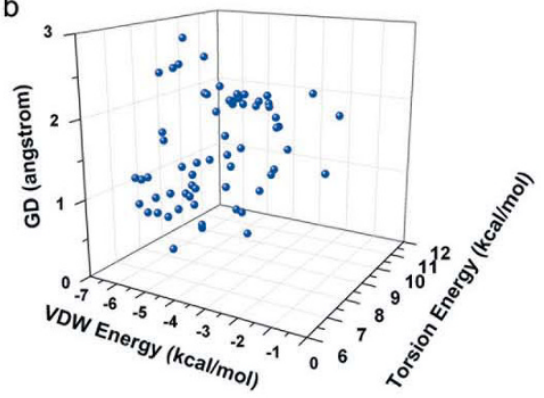

c

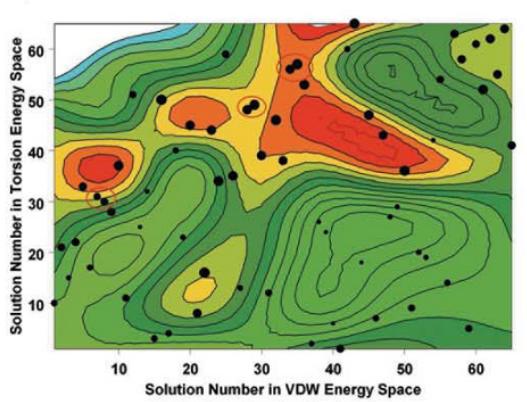

Figure 3

Distribution of rotatable bonds in the test set containing 329 molecular structures. 


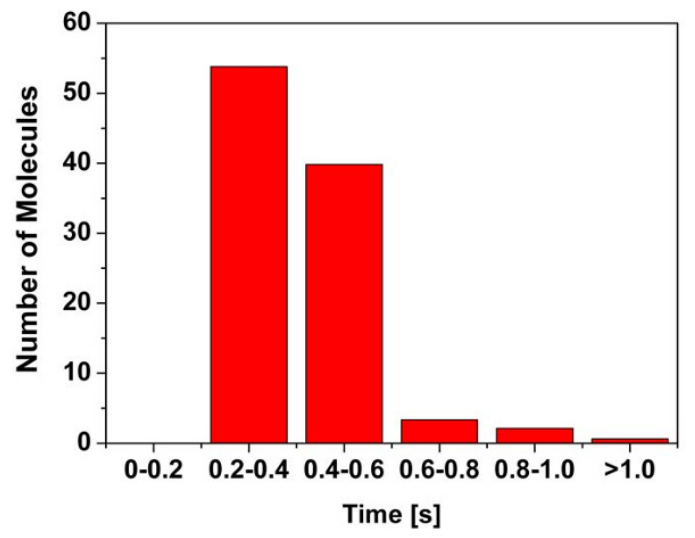

Figure 4

Distribution of CPU time used in MOEA procedure for all 329 conformer ensembles.

\section{Assesing the ability of reproducing bioactive conformations of Cyndi}

To evaluate Cyndi's capability of reproducing the bioactive crystal structures, the distribution of minimum RMSD values between best-fit conformers and crystal structures is shown in Figure 6 by plotting against the number of rotatable bonds of corresponding molecules. The minimum RMSD values are linearly correlated to the numbers of rotatable bonds of ligands with $R^{2}=0.68$, which is in accordance to the expectation that the predictive inaccuracy increases with the molecular flexibility. The average minimum RMSD value of total 329 conformational ensembles to their crystal bound conformers is $0.864 \pm$

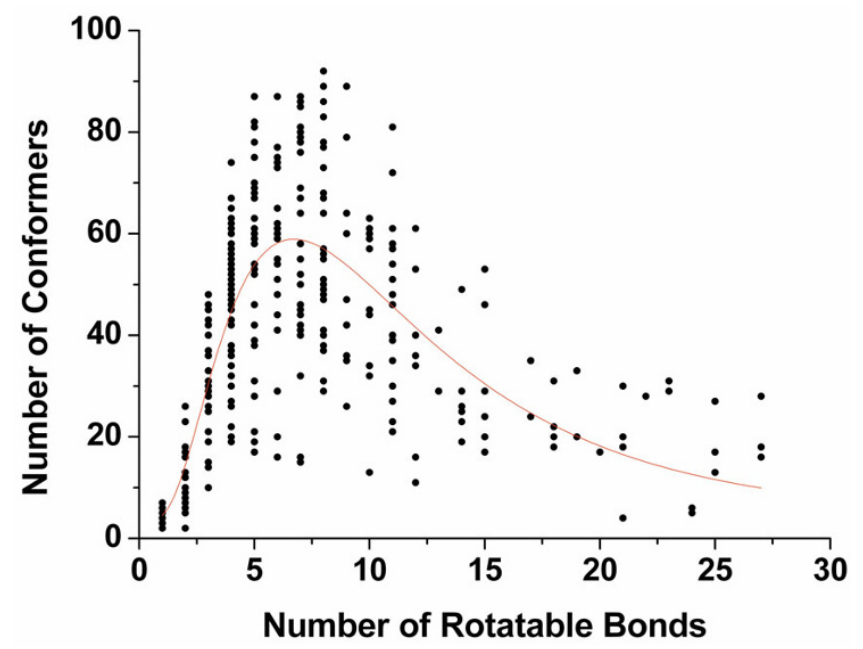

Figure 5

Number of generated conformers per molecule plotted against the number of the rotatable bonds. The observed skewed peak distribution trend is represented in the solid line.
$0.687 \AA$ for 3 -objective case $(0.831 \pm 0.601 \AA$ for 4 -objective case), and in addition, nearly $67.2 \%$ of the minimum RMSD values are below $1.0 \AA$ (93.6\% below $2.0 \AA)$. As a comparison, both the results of Cyndi with and without the optional objective (gyration radius) are compared with Balloon, and three conformation generation methods provided in Catalyst. Figure 7 shows the histogram of the cumulative distribution of the 329 molecules whose minimum RMSD to crystal conformers is within specific cutoff thresholds. It's apparent that the results of Cyndi are better or equal to those of the 4 methods considering that they share similar percentage of conformers within 2 $\AA$ threshold to the bioactive ones. With regard to the apparent potency of bioactive conformations reproduction $35.2 \%$ of the minimum RMSD of generated conformations are within $0.5 \AA$ intervals from the bioactive ones) as well as speedup advantage, Cyndi outperforms other methods both in accuracy and efficiency. Table A1 (see Additional file 2) lists more detailed comparisons of Cyndi and other methods for each molecule. The result of initial single 3D conformers generated by ChemAxon's Standardizer module is also tabulated in Table A1 (see Additional file 2) as reference.

Comparing with 3-objective case, additional objective of gyration radius slightly bettered the result (lowering the average minimum RMSD from $0687 \AA$ to $0.607 \AA$ ) and the number of minimum RMSD within 0-0.5 $\AA$ cutoff to the bioactive conformers increased from 107 to 116 . Essentially, considering the definition of gyration radius and essence of VDW energy, there would be certain overlapping between these two objectives on penalizing the steric bumped conformations, which has been observed (data not shown). This is part of the reason that gyration

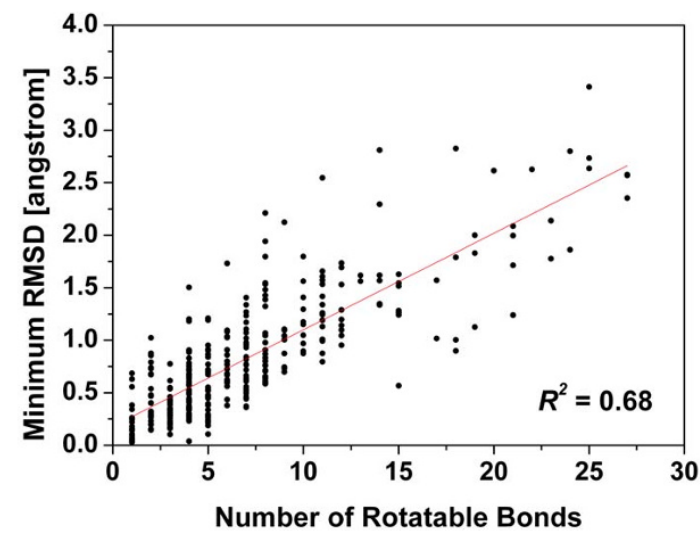

Figure 6

Minimum RMSD by superposition of generated conformers and crystal bound conformation plotted against the number of rotatable bonds. The observed linear trend is presented in solid line. 


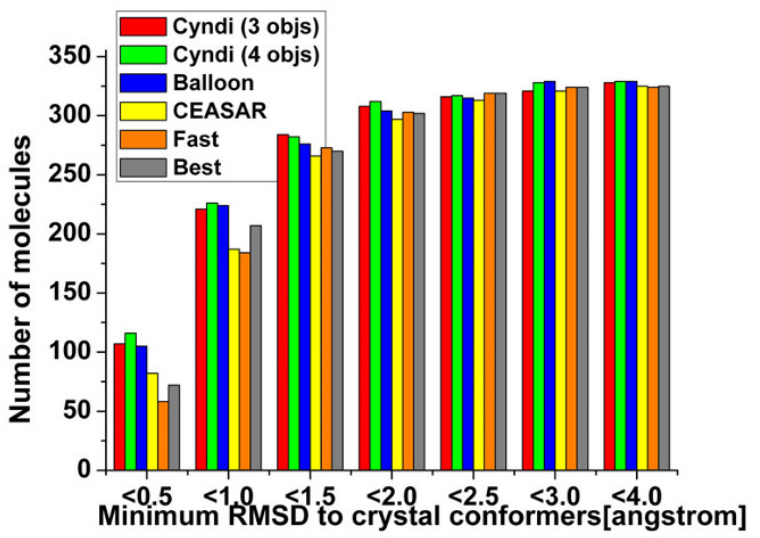

Figure 7

The distribution of best-fit conformers (defined by minimum RMSD to crystal structures) to 329 compounds for Cyndi, Catalyst Fast, Catalyst Best, Catalyst CEASAR and balloon. The best fit RMSD cumulative distributions for 329 molecules are compared among 6 methods: Cyndi (both with 3 and 4 objectives), Catalyst Fast, Catalyst Best, Catalyst CEASAR and Balloon. Note that Fast, Best and CEASAR failed to generate conformations for some molecules, as the entries labeled by "NA" in Table AI (see Additional file 2).

radius was formulated as an optional objective. Including gyration radius may improve the qualities of generated conformations for some flexible molecules, but it is not always the case. Actually the Pareto solutions in 4-objective space do not necessarily bear larger gyration radius because MOEA algorithm never optimizes towards the extremum of any single objective. Figure 8 depicts the alignments of best-fit conformers for two flexible molecules (PDB entries $\underline{1 \text { if8 }}$ and $\underline{1 \mathrm{sme}}$ ) generated with and without gyration radius objective. For 1 if 8 , the bioactive structure adopts a relatively extended conformer with a gyration radius of $5.250 \AA$; comparing with the conformer generated in 3-objective case, the minimum RMSD (to the bioactive one) of conformer generated by Cyndi with 4objective is reduced from $1.186 \AA$ to $0.501 \AA$ with gyration radius increasing from $4.830 \AA$ to $5.233 \AA$ (see Figure 8a); while for 1 sme, the minimum RMSD increases from $1.754 \AA$ to $2.569 \AA$ with gyration radius increasing from $6.093 \AA$ to $6.274 \AA$ for 3 -objective and 4-objective cases respectively (see Figure $8 \mathrm{~b}$ ). In fact, additional objective separates the solutions across more refined hyper grids on the Pareto frontiers so as to sample the conformational space more thoroughly. For linear molecules, including gyration radius may boost the procedure of conformational sampling if the bioactive conformers adopt more extended conformation; however, maximizing gyration radius in Cyndi may blur the unbiased sampling procedure for highly branched molecules (as 1sme in Figure a
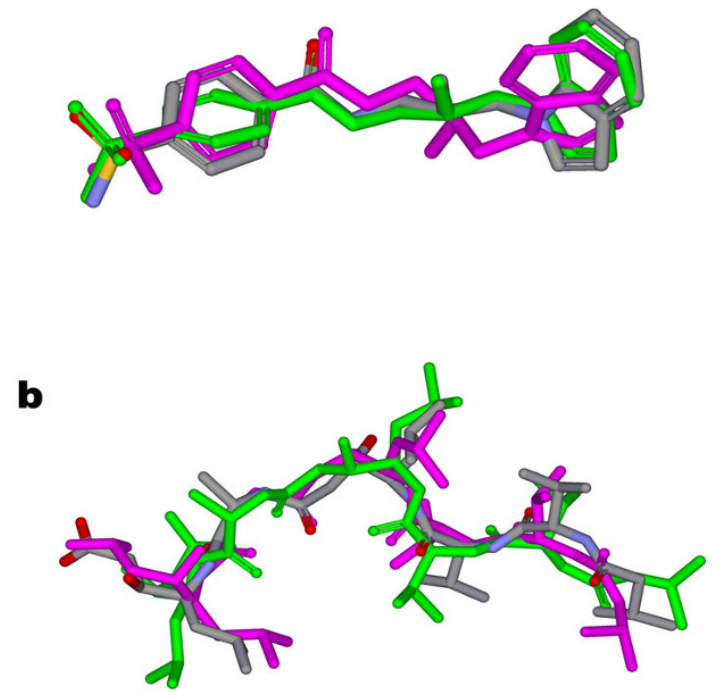

Figure 8

Comparing the performance of Cyndi with 3 objectives and 4 objectives. Exemplary alignments of conformers generated by Cyndi with 3 and 4 objectives: (a) IIF8 and (b) ISME. Only the best-fitted conformers to the bioactive structures are shown. The crystal structure conformations are coloured in terms of element (Carbon in grey; oxygen in red and nitrogen in blue), the conformers generated by Cyndi with 3 objectives are coloured in magenta and the ones with 4 objectives are in green.

8b) which may harbor multiple reasonable spatial extensions. Practically, because of the inherit irreproducibility flaw of evolution algorithm, one is advised to repeat several independent runs from diverse multiple random conformations both with and without gyration radius objective to sample the conformational space more thoroughly, as Table A2 (see Additional file 3) shows, the results from 3 independent runs of Cyndi outperformed that of single run to some extent.

\section{Assessment geometrical diversity of generated conformer ensembles}

The RMSD filter was applied to remove the conformational redundancy. To quantify the geometrical variation, agglomerative hierarchical clustering analysis was performed on the populated distance matrix via calculating the RMSD values between each pair of conformers. The resulted clustering dendrograms as well as corresponding reordered similarity heatmaps for four selected conformer ensembles are shown in Figure 9. In principle, if the conformers in the ensemble are totally geometrically different from each other, explicit non-overlapping diagonal blocks should emerge from the similarity matrix. The ligand of 1 apt (isovaleryl (iva)-val-val-lysta-o-et) is typically a peptide mimic with 3 peptide bonds and a total of 18 rotata- 
ble bonds. Since the stiffness induced by the peptide bonds (energy-constrained close to 0 and 180 degrees), the conformer ensemble is generally clustered into two major groups indicated by two large on-diagonal blocks, the members in each cluster are at least within $3.0 \AA$ RMSD threshold against each other (see Figure 9a). Each of the large clusters is divided into much smaller independent ones if we lower the dissimilarity threshold to 0.5 or $1.0 \AA$, indicating the generated conformers are geometrically dissimilar to each other at this level. Similar results have also been observed in the case of all other three molecules because they all bear one or more peptide bonds. Comparing with 1apt, more distinguishable small-size on-diagonal blocks are exemplified by ligand of $1 \mathrm{tmn}(\mathrm{N}-$ carboxymethyl dipeptide, 14 rotatable bonds) and that of 1epo (mor-phe-nle-chf-nme, 20 rotatable bonds) in Figures $9 \mathrm{~b}$ and $9 \mathrm{~d}$. The conformers are geometrically distinguishable with each other below the RMSD threshold of $2.0 \AA$, illustrating the generated conformers are highly distantly spread in conformational space. As for a less flexible case, the ligand of $8 \mathrm{gch}$ (gly-ala-trp tripeptide with 9 rotatable bonds) in Figure 9c exhibits a more blurred profile of clustering heatmap below the RMSD threshold of $2.0 \AA$. Allowing for the more compact conformational space formed by relatively small number of rotatable bonds, the RMSD threshold for dissimilarity should be lowered by reducing the scaling factor in GD filter.

Theoretically, the generated conformers should maximize diversity by distinguishing geometrically with each other completely. Practically, this goal can not be achieved because for a molecule bearing middle level flexibility (like 1epo), the energy-favoured accessible areas are limited with respect to broadly sampled conformational space. Herein many conformers have to be discarded which failed to pass the low-energy filter despite their geometrical dissimilarity. The RMSD threshold in the RMSD filter increases with molecular flexibility, and for those molecules with moderate flexibility (with number of rotatable bonds up to 15), the threshold is about $0.5 \AA$ with the scaling parameter set to 0.1. From the observation of the clustering heatmaps, most of the conformers are geometrically different from each other at the threshold level of $0.5 \AA$ A considering the corresponding on-diagonal blocks are small-size and non-overlapped (filled by deepest red colour). When increasing the threshold, the areas of on-diagonal blocks become larger and the corresponding borders turn out fuzzier. Cyndi employs a scalable RMSD threshold instead and achieves a good compromise to generate limited number (usually no more than 100 averagely) of diverse conformers.

It may be argued that the generated conformers can be biased geometrically to mimic the starting conformation because GD objective reflects essentially minimized RMSD between the individuals and initial conformation. Actually, different from single objective GA in which the population evolves towards the extremum in the single objective space exclusively, the optimization essential of Cyndi is to generate a non-dominant solution set in the hyperspace of multiple objectives because no single or a few objectives can exclusively harness the optima towards the extrema in single or parts of objective space. For the Pareto optimal conformers in the final archive, most of them are geometrically distant from the initial conformers because they are favourable in other objective spaces such as VDW and torsion energy. Namely the objectives divide the objective hyperspace into grids with the size of corresponding $o$ values and the Pareto solutions scatters across those non-dominant grids consisting of Pareto frontier.

Currently, Cyndi only supports generating conformer ensemble from an initial 3D conformation, and moreover, since Cyndi merely perturbs the dihedral torsions in optimization procedure, it is not compulsive but highly recommended starting from the conformer converted by the 2D-to-3D conversion toolkits or the one energy-minimized to local minimum with appropriate force field to ensure the generated conformers bear reasonable bond lengths and bond angles. The work of integrating the 1Dto-3D and 2D-to-3D features to perfect Cyndi in handling the problem of conformation generation is under progress.

\section{Conclusion}

A new conformation generation method Cyndi was designed based on a fast and robust MOEA theory. Using multiple objectives controlling energy accessibility as well as geometric diversity, Cyndi is capable of searching the conformational space in nearly constant time and sampling the Pareto frontier on which both energy and diversity features are favoured. Three independent objectives (VDW energy, torsion energy and geometrical dissimilarity) are employed and tested against 329 ligands with available crystal determined conformations, the resulted minimum RMSD to bioactive conformers amounts to $0.864 \AA$ in average. The objectives can be implemented easily so it's very simple to customize Cyndi to generate different conformation ensembles which are pareto optimals scattering in the hyperspace formed by different objectives.

\section{Methods}

\section{Overview of Multi-objective Evolution Algorithm}

In real life, most optimization problems involve multiple objectives (possibly in conflict), which should be satisfied simultaneously. Generally, optimization with single objective often results in "optimal" solutions against only 

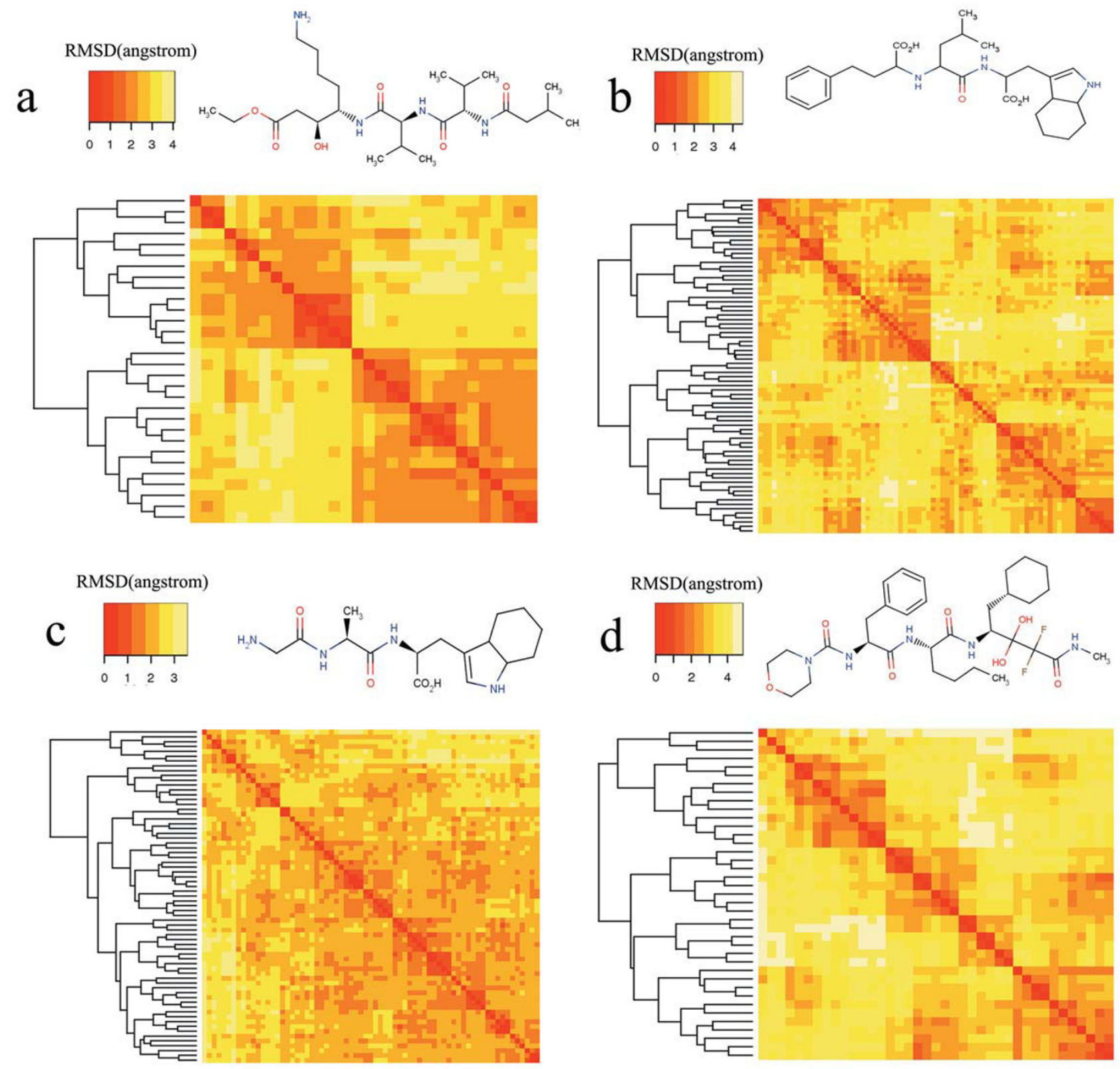

\section{Figure 9}

Clustering analyses of generated conformers for $\mathbf{4}$ selected molecule structures. The dendrograms and reordered similarity heatmaps represent the results of the complete-linkage clustering, applying to the distance matrix populated via computing the global RMSD values between each pair of conformers in the ensembles. The blocks in the heatmaps are coloured according to the legend which represents RMSD value as colour bins. The structures for a) IAPT, b) ITMN, c) 8GCH, and d) IEPO are presented. 
one objective but unacceptable with respect to the other ones. To solve this trade-off problem, multiple instead of single reasonable solutions should be reserved, incorporating multiple objectives optimized simultaneously. A solution is said to be Pareto optimal if it is not dominated by any other solutions in the solution space, which cannot be improved with respect to any objective without worsening at least one of other objectives. The set of all feasible non-dominated solutions is referred to as the non-dominated or Pareto optimal solutions and the Pareto frontier can be mapped out by ranking the fitness of the Pareto optimal solutions in the search space, see references [4345] for more details.

Through the operation on the population of individuals with inherent parallel mechanism, GA or EA is suitable to solve multi-objective optimization problems. The crossover operator may exploit structures of good solutions with respect to different objectives to create new non-dominated solutions in unexplored parts of Pareto front. There are many MOGA and MOEA methods tailored for different practical problems, and generally speaking they are merely different with respect to fitness assignment procedure, elitism, or diversification approaches [46].

We adopt $o$-MOEA method, a robust algorithm based on the concept of $o$-domination developed by Deb et al. $[47,48]$ as our conformational searching engine. In $o$ MOEA, two populations co-evolve, one is the general evolving population $P(t)$ identical to those used in singleobjective GA, the other is the "archive" population $A(t)$ serving as the collection of Pareto solutions generated in the $t$ th generation. $A(0)$ is the $o$-non-dominated solution set of $\boldsymbol{P}(\mathbf{0})$ after initialization. The crossover operator is applied to two individuals randomly selected from $P(t)$ and $A(t)$, respectively, and each new child is compared with the individuals in the two populations by the o-domination method to check if it would be accepted or not. Each individual in the archive is defined as identification array $\mathbf{B}$ as following:

$$
B_{j}(f)= \begin{cases}\left\lfloor\left(f_{j}-f_{j}^{\min }\right) / \varepsilon_{j}\right\rfloor, & \text { for minimizing } f_{j} . \\ {\left[\left(f_{j}-f_{j}^{\min }\right) / \varepsilon_{j}\right],} & \text { for maximizing } f_{j .}\end{cases}
$$

where $f_{j}^{\min }$ is the minimum probable value of the $j$ th objective function and $o_{j}$ is the user-definable tolerance between two objective values. Identification array divides the whole $j$ th objective function space into the hyper- boxes with the size of $o_{j}$. If the identification array of the new child dominates any archive individual, then the dominated individual is substituted by the new child; otherwise the new child is discarded. If none of the two conditions is satisfied, the new child is a $o$-non-dominated solution. Following criteria are used to handle the new child:

1. When the new child and one archive individual share the same identification array $\mathbf{B}$, namely they are in the same box, the new child would only be accepted either if it dominates the archive individual or it is nearer to $\mathbf{B}$.

2. When the new child doesn't share one identification array $\mathbf{B}$ with any of archive individuals, the new child would be accepted.

In this way, the distribution of Pareto optimal solution set is attained under the condition that each box of the Pareto frontier can only be occupied by one solution. The number of Pareto optimal solutions can be restrained by scaling the $o$ value.

Similarly, the selection procedure in the population $P(t)$ is also determined by epsilon domination. If the new child dominates one or more individuals in $P(t)$, the new child would substitute one of such individuals randomly, or else it should be discarded. If they are non-dominated by each other, one of them would be substituted by the other randomly to fix the size of the population.

\section{Details of MOEA in Cyndi}

\section{I Genome Encoding}

Similar to the encoding scheme used in traditional single objective GA operated on molecules, namely the molecules are divided into a set of rigid fragments linked by several rotatable single bonds, Cyndi encodes the genome into a vector of real numbers within the interval of $[0,2 \pi)$, which represents the torsion angle each rotatable bond to be rotated during the conformation generation. The phenotypes, namely the conformers, are easily translated through rotating the single bond by the values coded in each gene. There have been some reports declaring that reducing the continuous torsion space into discrete one (using torsion grid or biasing the torsion value towards the favourable ranges discovered through mining torsion space) may boost convergence [15], however, this assumption may cause missing some conformational significant areas due to the limited accessible information, for this reason, we still manipulate the genomes in continuous torsion space. 


\subsection{Optimization Objective Functions}

Two terms of potential energy calculated with Tripos force field, VDW and torsion energy, are taken as parts of the objectives for separating the solutions in energy space, during which both bond lengths and bond angles are frozen. The torsion term is directly subjected to the geometric variations during the evolution, consequently it is selected as one of the objectives; the second objective is VDW energy in that it increases exponentially with intensive steric bumps so as to penalize the "ugly" conformers. The format of VDW term is a conventional Lenard-Jones 12-6 function defined in Tripos force field and no cutoff is employed regarding the small size of most drug-size molecules. Since all the energy calculations are performed without considering solvent effects, electrostatic term is also discarded to avoid abnormal compact conformations induced by intra-molecular interaction such as internal hydrogen bonds and salt bridges.

The profile of energy landscape is often described as a zigzagged hypersurface. Two geometrically similar conformers may locate quite distant in energy hypersurface but converge to one minimum after energy optimization. Therefore a mechanism is necessary to promote geometric diversity in the population of evolving conformers; the geometric dissimilarity (GD) between each individual and the input conformation is set as the third objective, to control the geometrical distribution of the Pareto optimal solutions. The GD calculation is formulated as eq. (2):

$$
G D=\sqrt{\frac{\sum_{i=1}^{N}\left\|x_{\text {indiv }, i}-x_{\text {input }, i}\right\|^{2}}{N}}
$$

where $x_{\text {indiv, } i}$ and $x_{\text {input, } i}$ are the positions of the $i$ th heavy atom in each individual and the input conformer. Here only the positions of the heavy atoms are considered in GD calculation.

Previous study indicated most of the bioactive conformers tend to adopt relatively extended geometries [16], in light of this important observation, Cyndi provides an optional objective to direct the evolution towards generating conformers with different geometrical extension degree. Similar to the work by Izrailev et al., the objective that quantifies the degree of extension is formulated by calculating the gyration radius for each molecule, as defined in eq.(3):

$$
R_{g}=\sqrt{\frac{\sum_{i=1}^{n} m_{i}|| \mathbf{x}_{i}-\overline{\mathbf{x}} \|^{2}}{\sum_{i=1}^{n} m_{i}}}
$$

where $\mathbf{x}_{i}$ and $m_{i}$ are the position and mass, respectively, of the $i$ th atom; $\overline{\mathbf{x}}$ is the geometrical centre of the molecule. This objective may improve the conformational diversity if we have no idea about whether the bioactive conformer is compact or extended.

The multi-objective optimization model of Cyndi consists of a set of $n$ parameters (design variables), a set of $l$ objective functions, and a set of $m$ constraints. Objective functions and constraints are functions of the design variables. It can be formulated mathematically as follows:

$$
\begin{array}{ll}
\min & y=f(x)=\left(f_{1}(x), f_{2}(x), \ldots, f_{1}(x)\right) \\
\text { s.t. } & \boldsymbol{g}(\boldsymbol{x})=\left(g_{1}(\boldsymbol{x}), g_{2}(x), \ldots, g_{m}(\boldsymbol{x})\right) \leq 0 \\
\text { where } & \boldsymbol{x}=\left(x_{1}, x_{2}, \ldots, x_{n}\right) \in X \\
& \boldsymbol{y}=\left(y_{1}, y_{2}, \ldots, y_{1}\right) \in Y
\end{array}
$$

where $x$ is the design vector $x=\left\{T_{b 1},<, T_{b n}\right\}^{\mathrm{T}}$, in which $T_{b 1}$, $<, T_{b n}$ are the torsion angles of the $n$th rotatable bonds. Accordingly, the constraints for the design variables $(g(x) s)$ can be represented as

$$
0 \leq T_{b 1},<, T_{b n}<2 \pi
$$

$y$ is the objective vector, which consists of VDW term $f_{1}(x)$, torsion score $f_{2}(x)$, and the diversity of geometry $f_{3}(x)$ or $f_{4}(x)$, respectively. Note that the maximal problem for GD or gyration radius of each conformation is converted to minimal problem through reciprocal to ensure the most conformational distribution in geometric space. The maximal $X$ is denoted as the decision space, and $Y$ is denoted as the objective space.

The objective functions of Cyndi are easy to extend and customize without modifying kernel of the algorithm. By integrating other objectives as constraints, such as the interaction energy of ligand with the binding receptor or pharmacophore model matching, Cyndi would evolve to facilitate structure or ligand based virtual screening easily.

\subsection{Archive Post Processing}

The final archive contains the optimal solutions on Pareto frontier which have been separated by the objectives and are non-dominant to each other. However, the conformers in the archive may be energy-unfavourable or geometrically crowded because they may be suboptimal in the single objective case. Therefore, the final set of conformations is the pruned result of archive members by userdefinable energy and RMSD filters.

The conformational energy and geometry are optimized using the conjugated gradient (CG) method with Tripos force field, and all energy terms excluding electrostatic 
term and torsion term lest inner electrostatic interaction and torsion angle perturbation undermine generated conformation. After energy minimization, following energy cutoff is applied to discard those conformers beyond the energy window:

$$
E_{\text {cutoff }}=E_{w}+k \times N_{\text {rot }}
$$

where $E_{w}$ is a user-definable energy cutoff (default is 20 $\mathrm{kcal} / \mathrm{mol}$ ), $N_{\text {rot }}$ is the number of rotatable bonds of current molecule and $k$ is the scaling parameter (user-definable and the default value is 0.5). Similar to Balloon's approach, such energy cutoff takes into account of the effect imposed by molecular flexibility and reflects the observed dependence between the local minima and number of rotatable bonds of bioactive conformation [49]. Only those conformers within the energy threshold are labelled as energy-favoured and survived to subsequent RMSD filter.

The conformers survived from the energy filter are submitted to a RMSD filter to remove geometrical duplicates. We adopted the filter scheme defined in Catalyst [50]. Conformers are compared with each other in terms of RMSD and any conformer within the RMSD tolerance (vide infra) of another conformer is discarded:

$$
R M S D_{t o l}=0.1+c \times \sqrt{1+N_{\text {rot }}}
$$

where $c$ is a user-definable scaling parameter, and $N_{\text {rot }}$, again, is the number of rotatable bonds. The involvement of molecular flexibility can efficiently reduce the geometrical redundancy for highly flexible molecules.

\section{Testing of Cyndi}

The ability for conformation generation of Cyndi was tested against a data set containing 329 drug-sized molecules whose bioactive conformations were extracted from the PDB database (see Additional file 1). This is a combined set of part of CCDC/Astex subset [51,52] used in Balloon's validation and the subset used by Izrailev et.al. $[53,54]$. To avoid the influences on the generation of conformers from existing crystal structures, single 3D conformer was regenerated for each molecule from 2D structures using the Standardizer module of ChemAxon package [55].

Cyndi test run was performed on each of the molecules in the test set with 200 populations and 200 generations. The probabilities for crossover and mutation operation were set to 0.85 and 0.1 , respectively. The relatively larger mutation probability was designated to sample the conformational space more broadly by mutating the existing conformers more frequently. The epsilon values for the four objectives (VDW energy, torsion energy, GD value, and gyration radius) were set as $20 \mathrm{kcal} / \mathrm{mol}, 5 \mathrm{kcal} / \mathrm{mol}$, $0.2 \AA$ and $0.1 \AA$, respectively. The maximum iteration for post processing CG minimization was set to 100, and the convergence criterion based on gradient RMS was set to $0.1 \mathrm{kcal} \cdot \mathrm{mol}^{-1} \cdot \AA^{-1}$. No initial optimization against the input conformers was applied and the input conformers were discarded from the final conformer ensembles. Other parameters were set to the default values mentioned previously.

To make a comparison, the Pentium4 processor optimized version of Balloon (version 0.6.6.4641) [56] as well as three conformer generation methods (Catalyst Best, Catalyst Fast and CEASAR) in DS2.0 [57-60] were run against the same test. All default parameters were applied and the maximum number of generated conformers was limited to 100. The initial single 3D conformers generated by Standardizer of ChemAxon were also retained for further comparison (see Table A1 in Additional file 2).

To make an impartial comparison, the minimum RMSD values between the crystal bound conformers and the conformers generated by all methods were calculated with a third-party program, Vrms 1.0, which is an RMSD calculator removing artificial differences caused by symmetry [61]. In this study, a conformation is considered to be successfully reproduced if the RMSD value is less than $0.5 \AA$, as compared to the original X-ray structure. Balloon, Cyndi, Vrms and DS 2.0 were all run on a workstation with an Intel Pentium Dual Core Processor (2.66 GHz).

Hierarchical clustering was applied to analyze the conformational diversity of each conformer ensemble generated by Cyndi. Vrms calculated RMSD values between each pairs of conformers were used to populate a distance matrix, which was then analyzed by applying complete linkage agglomerative hierarchical clustering. The clustering results were visualized by dendrograms and reordered heatmaps of the distance matrix.

\section{Authors' contributions}

XL designed and validated Cyndi method and also contributed to analysis and data interpretation. FB contributed to the design of method. SO contributed to method validation. HL conceived the idea of the MOEA in conformation generation and co-drafted the manuscript with XL. $\mathrm{HL}, \mathrm{XW}$ and HJ participated in Cyndi's design, provided direction for its development and revised the subsequent drafts of this manuscript. All authors read and approved the final manuscript. 


\section{Additional material}

\section{Additional File 1}

The pre-compiled version of Cyndi (Windows, 32-bit $\times 86$ ), parameter input file, Tripos force field parameter file and the test set of 329 high-resolution and drug-concerned small molecules determined from X-ray structures in mol2 format.

The pre-compiled version of Cyndi (Windows, 32-bit $\times 86$ ), parameter input file, Tripos force field parameter file and the test set of 329 highresolution and drug-concerned small molecules determined from $X$ ray structures in mol2 format.

Click here for file

[http://www.biomedcentral.com/content/supplementary/14712105-10-101-S1.gz]

\section{Additional File 2}

Detailed calculation results for best-fit conformers of 329 crystal structures by Cyndi and other five conformation generation programs.

Detailed calculation results for best-fit conformers of 329 crystal structures by Cyndi and other five conformation generation programs. Click here for file

[http://www.biomedcentral.com/content/supplementary/14712105-10-101-S2.doc]

\section{Additional File 3}

Detail comparison generated conformers from 3 independent runs against 84 structures.

Detail comparison generated conformers from 3 independent runs against 84 structures. Distribution of the conformers in 3-objective space from 3 independent runs of 1 sme viewing along $x, y, z$ axis respectively. Click here for file

[http://www.biomedcentral.com/content/supplementary/14712105-10-101-S3.doc]

\section{Acknowledgements}

This work was supported by the National Natural Science Foundation of China (grants 20803022, 30672539 and 2072 1003), the Shanghai Committee of Science and Technology (Grant 07dz22004), the $863 \mathrm{Hi}-$ Tech Program of China (Grant 2007AA02Z304), the International Collaboration Project of China (Grant 2007DFB30370) and the National Basic Research Program of China (Grants 2009CB9I850I and 2009CB9/8502). H.L. was also supported by Knowledge Innovation Program of the Chinese Academy of Sciences (Grant SIMM0709QN-09). The authors thank Professor Deb for providing MOEA source code, Dr. Vainio for providing Balloon, Dr. Michael J. Potter from VeraChem for Vrms. We also thank Dr. Jiabo Li and Dr. Deqiang Zhang from Accelrys for their helpful discussions and suggestions.

\section{References}

I. Leach AR: A Survey of Methods for Searching the Conformational Space of Small and Medium-Sized Molecules. In Reviews in Computational Chemistry Volume 2. Edited by: Lipkowitz KB, Boyd DB. New York, VCH Publishers; 1991:1-55.

2. Lorber DM, Shoichet BK: Flexible ligand docking using conformational ensembles. Protein Sci 1998, 7:938-950.

3. Jackson RM: Q-fit: a probabilistic method for docking molecular fragments by sampling low energy conformational space. J Comput-Aided Mol Des 2002, 16:43-57.
4. Lee K, Czaplewski C, Kim SY, Lee J: An efficient molecular docking using conformational space annealing. J Comput Chem 2005, 26:78-87.

5. Bronowska A, Les A, Mazgajska M, Chilmonczyk Z: Conformational analysis and pharmacophore design for selected I-(2pyrimidinyl) piperazine derivatives with sedative-hypnotic activity. Acta Pol Pharm 200I, 58:79-86.

6. Kristam R, Gillet VJ, Lewis RA, Thorner D: Comparison of conformational analysis techniques to generate pharmacophore hypotheses using catalyst. J Chem Inf Model 2005, 45:46I-476.

7. Thimm M, Goede A, Hougardy S, Preissner R: Comparison of 2D similarity and 3D superposition: Application to searching a conformational drug database. J Chem Inf Comput Sci 2004, 44: $1816-1822$.

8. Goede A, Dunkel M, Mester N, Frommel C, Preissner R: SuperDrug: a conformational drug database. Bioinformatics 2005, 21:1751-1753.

9. Gopalakrishnan K, Sheik SS, Ranjani CV, Udayakumar A, Sekar K Conformational Angles DataBase (CADB-3.0). Protein Pept Lett 2007, 1 4:665-668.

10. Martinek TA, Otvos F, Dervarics M, Toth G, Fulop F: Ligand-based prediction of active conformation by 3D-QSAR flexibility descriptors and their application in 3+3D-OSAR models. Med Chem 2005, 48:3239-3250.

II. Yuan H, Petukhov PA: Improved 3D-QSAR CoMFA of the dopamine transporter blockers with multiple conformations using the genetic algorithm. Bioorg Med Chem Lett 2006, 16:6267-6272.

12. Dezi C, Brea J, Alvarado M, Ravina E, Masaguer CF, Loza MI, Sanz F, Pastor M: Multistructure 3D-QSAR studies on a series of conformationally constrained butyrophenones docked into a new homology model of the 5-HT2A receptor. J Med Chem 2007, 50:3242-3255.

13. Haigh JA, Pickup BT, Grant JA, Nicholls A: Small Molecule ShapeFingerprints. J Chem Inf Model 2005, 45:673-684.

14. Teague SJ: Implications of protein flexibility for drug discovery. Nature Reviews Drug Discovery 2003, 2:527-54I.

15. Smellie A, Stanton R, Henne R, Teig S: Conformational analysis by intersection: CONAN. J Comput Chem 2003, 24:10-20.

16. Diller DJ, Merz KM Jr: Can we separate active from inactive conformations? J Comput-Aided Mol Des 2002, 16:105-II 2.

17. Kirchmiar J, Laggner C, Wolber G, Langer T: Comparative analysis of protein-bound ligand conformations with respect to catalyst's conformational space subsampling algorithms. J Chem Inf Model 2005, 45:422-430.

18. Boström J, Norrby P, Liljefors T: Conformational energy penalties of protein-bound ligands. J Comput Aided Mol Des 1998, I 2:383-396.

19. Boström J: Reproducing the conformations of protein-bound ligands: A critical evaluation of several popular conformational searching tools. I Comput Aided Mol Des 200I, I 5: I I37- II 52.

20. Boström J, Greenwoodb JR, Gottfriesa J: Assessing the performance of OMEGA with respect to retrieving bioactive conformations. J Mol Graph Model 2003, $21: 449-462$.

21. Budin N, Majeux N, Tenette-Souaille C, Caflisch A: Structurebased ligand design by a build-up approach and genetic algorithm search in conformational space. J Comput Chem 200I, 22: 1956-1970.

22. De Magalhaes CS, Barbosa HJC, Dardenne LE: A genetic algorithm for the ligand-protein docking problem. Genet Mol Biol 2004, 27:605-610

23. Jones G, Willett P: Docking and Superimposition of Flexible Molecules Using a Genetic Algorithm. Abstr Paper Am Chem Soc 1995, 209: 144

24. Jones G, Willett P, Glen RC: A genetic algorithm for flexible molecular overlay and pharmacophore elucidation. / ComputAided Mol Des 1995, 9:532-549.

25. Judson RS, Jaeger EP, Treasurywala AM, Peterson ML: Conformational Searching Methods for Small Molecules .2. Genetic Algorithm Approach. J Comput Chem 1993, I 4:1407-I4I4.

26. Cao TC, $\mathrm{Li}$ TH: A combination of numeric genetic algorithm and tabu search can be applied to molecular docking. Comput Biol Chem 2004, 28:303-3I2. 
27. Li HL, Li CL, Gui CS, Luo XM, Chen KX, Shen JH, Wang XC, Jiang $H L$ GAsDock: a new approach for rapid flexible docking based on an improved multi-population genetic algorithm. Bioorg Med Chem Lett 2004, I 4:467I-4676.

28. Mekenyan O, Dimitrov D, Nikolova N, Karabunarliev S: Conformational coverage by a genetic algorithm. J Chem Inf Comput Sci 1999, 39:997-1016.

29. Morris GM, Goodsell DS, Halliday RS, Huey R, Hart WE, Belew RK, Olson Al: Automated docking using a Lamarckian genetic algorithm and an empirical binding free energy function. J Comput Chem 1998, 19:1639-1662.

30. Pavlov T, Todorov M, Stoyanova G, Schmieder P, Aladjov H, Serafimova R, Mekenyan O: Conformational coverage by a genetic algorithm: Saturation of conformational space. J Chem Inf Model 2007, 47:85I-863.

31. Taha MO, Qandil AM, Zaki DD, AIDamen MA: Ligand-based assessment of factor $\mathrm{Xa}$ binding site flexibility via elaborate pharmacophore exploration and genetic algorithm-based QSAR modeling. Eur J Med Chem 2005, 40:701-727.

32. Lameijer EW, Kok JN, Back T, ljzerman AP: The molecule evaluator: An interactive evolutionary algorithm for the design of drug-like molecules. J Chem Inf Model 2006, 46:545-552.

33. Valerie JG: Applications of Evolutionary Computation in Drug Design. Struct Bond 2004, I I 0: I33-I52.

34. Grosdidier A, Zoete V, Michielin O: EADock: docking of small molecules into protein active sites with a multiobjective evolutionary optimization. Proteins 2007, 67:1010-1025.

35. Cottrell S], Gillet VJ, Taylor R: Incorporating partial matches within multi-objective pharmacophore identification. J Comput-Aided Mol Des 2006, 20:735-749.

36. Cottrell SJ, Gillet V], Taylor R, Wilton DJ: Generation of multiple pharmacophore hypotheses using multiobjective optimisation techniques. J Comput-Aided Mol Des 2004, I 8:665-682.

37. Gillet V], Khatib W, Willett P, Fleming PJ, Green DV: Combinatorial library design using a multiobjective genetic algorithm. J Chem Inf Comput Sci 2002, 42:375-285.

38. Nicolotti O, Gillet VJ, Fleming PJ, Green DV: Multiobjective optimization in quantitative structure-activity relationships: deriving accurate and interpretable QSARs. J Med Chem 2002, 45:5069-5080.

39. Vainio MJ, Johnson MS: Generating conformer ensembles using a multiobjective genetic algorithm. J Chem Inf Model 2007, 47:2462-2474.

40. Shoichet BK, Leach AR, Kuntz ID: Ligand solvation in molecular docking. Proteins 1999, 34:4-I6.

4I. Berman HM, Westbrook J, Feng Z, Gilliland G, Bhat TN, Weissig H, Shindyalov IN, Bourne PE: The Protein Data Bank. Nucleic Acids Res 2000, 28:235-242.

42. Epsilon-MOEA [http://www.iitk.ac.in/kangal/codes.shtml]

43. Zitzler E, Deb K, Thiele L: Comparison of multiobjective evolutionary algorithms: empirical results. Evol Comput 2000, 8:173-195.

44. Coello CA: An updated survey of GA-based multiobjective optimization techniques. ACM Comput Surv 2000, 32:109-143.

45. Deb K: Multi-Objective Optimization using Evolutionary Algorithms. Chichester, UK; John Wiley \& Sons; 2001.

46. Konak A, Coit DW, Smith AE: Multi-objective optimization using genetic algorithms: A tutorial. Reliab Eng Syst Saf 2006, 91:992-1007.

47. Deb K, Mohan M, Mishra S: A fast multi-objective evolutionary algorithm for finding well-spread Pareto-optimal solutions. In KanGal Rep No.2003002, Indian Institute of Technology, Kanpur, India; 2003.

48. Laumanns M, Thiele L, Deb K, Zitzler E: Combining convergence and8diversity in evolutionary multiobjective optimization. Evol Comput 2002, 10:263-282.

49. Perola E, Charifson PS: Conformational analysis of drug-like molecules bound to proteins: An extensive study of ligand reorganization upon binding. J Med Chem 2004, 47:2499-25I 0.

50. Catalyst [http://www.accelrys.com/]

5I. Hartshorn MJ, Verdonk ML, Chessari G, Brewerton SC, Mooij WT, Mortenson PN, Murray CW: Diverse, high-quality test set for the validation of protein-ligand docking performance. J Med Chem 2007, 50:726-74I.
52. Nissink JW, Murray C, Hartshorn M, Verdonk ML, Cole JC, Taylor R: A new test set for validating predictions of protein-ligand interaction. Proteins 2002, 49:457-47I.

53. Agrafiotis DK, Gibbs AC, Zhu F, Izrailev S, Martin E: Conformational sampling of bioactive molecules: a comparative study. J Chem Inf Model 2007, 47: I067-I086.

54. Izrailev S, Zhu F, Agrafiotis DK: A distance geometry heuristic for expanding the range of geometries sampled during conformational search. J Comput Chem 2006, 27:1962-1969.

55. Standerdizer [http://www.chemaxon.com/product/standard izer.html]

56. Balloon [http://web.abo.fi/ mivainio/balloon]

57. Smellie A, Kahn SD, Teig SL: Analysis of conformational coverage. I. Validation and estimation of coverage. J Chem Inf Comput Sci 1995, 35:285-294.

58. Smellie A, Kahn SD, Teig SL: Analysis of conformational coverage. 2. Applications of conformational models. J Chem Inf Comput Sci 1995, 35:295-304.

59. Li J, Ehlers T, Sutter J, Varma-O'brien S, Kirchmair J: CAESAR: a new conformer generation algorithm based on recursive buildup and local rotational symmetry consideration. J Chem Inf Model 2007, 47:1923-1932.

60. Discovery Studio [http://www.accelrys.com/]

61. Vrms [http://www.verachem.com/vrms.html]
Publish with Biomed Central and every scientist can read your work free of charge

"BioMed Central will be the most significant development for disseminating the results of biomedical research in our lifetime. "

Sir Paul Nurse, Cancer Research UK

Your research papers will be:

- available free of charge to the entire biomedical community

- peer reviewed and published immediately upon acceptance

- cited in PubMed and archived on PubMed Central

- yours - you keep the copyright
BioMedcentral 